\title{
ROBUST REPETITIVE FEEDBACK CONTROL OF A THREE-PHASE GRID CONNECTED INVERTER
}

\author{
M. Jamil, S. M. Sharkh, M. Abusara, R. J. Boltryk \\ School of Engineering Sciences,University of Southampton, \\ Highfield,Southampton SO17 1BJ,United Kingdom. \\ Tel +44 2380 594641,Fax +442380 593053 \\ Email: mj2p07@soton.ac.uk
}

Keywords: Distributed Generator (DG), Grid Connected Converters, Current Control, Repetitive Controller (RC)

\begin{abstract}
This paper discusses the design of a repetitive feedback controller for a grid-connected two-level three-phase voltagesource inverter connected between a DC source and the grid through an LCL filter. The controller incorporates a classical two loop feedback of the output current and the capacitor current in addition to a repetitive feedback loop. The results show that the proposed technique improves the steady state error and the total harmonic distortion of output current in presence of utility harmonics.
\end{abstract}

\section{Introduction}

Pulse width modulation (PWM) voltage source inverters (VSI) similar to that shown in Fig.1 are commonly used to connect distributed generator (DG) systems such as micro combined heat and power (CHP) and renewable energy sources to an AC grid or local loads. They convert DC from photovoltaic generators, batteries, fuel cells or variable frequency AC from wind and marine turbine into 50/60 Hz AC power. The output current of the converter should meet the total harmonic distortion (THD) standards in the presence of grid harmonics [1, 2]. This is commonly achieved using active feedback control of the current injected into the grid.

Alternative control strategies and structures have been used for grid-connected inverters such as deadbeat control [3], optimal control [4], state-feedback [5], sliding mode [6] and resonant controllers [7], in addition to PID and classical compensators. It is also common to use the $d-q$ transformation [8]. The objective of these controllers is to increase the outer loop gain, and, hence improve disturbance rejection. But most of these controllers tend to suffer from relatively low loop gain at the fundamental frequency and its harmonics and hence tend to have poor disturbance rejection which results in poor output current THD if the grid voltage THD is relatively high. A better controller is required with high gain at the harmonic frequencies of interest.

Repetitive feedback based control techniques have the potential to improve the THD quality of the converter output by effectively increasing the loop gain at the fundamental frequency and its harmonics [9]. The effectiveness of repetitive control in terms of eliminating harmonic distortion in a voltage source inverter operating has been demonstrated in several publications [9-15].

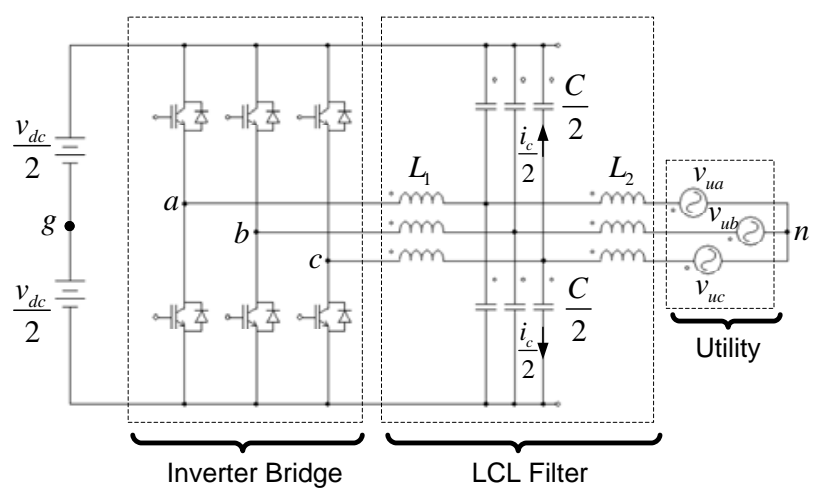

Fig. 1: Three Phase Grid Connected Inverter with LCL Filter

\begin{tabular}{|c|c|}
\hline Parameter & Value \\
\hline Utility Phase voltage & $230 \mathrm{~V}$ (rms) \\
\hline DC Link Voltage & $800 \mathrm{~V} \mathrm{dc}$ \\
\hline Inductor $L_{1}$ & $350 \mu \mathrm{H}$ \\
\hline Inductor $L_{2}$ & $50 \mu \mathrm{H}$ \\
\hline Capacitance $C$ & $22.5 \mu \mathrm{F}$ \\
\hline Switching Frequency & $10 \mathrm{KHz}$ \\
\hline
\end{tabular}

Table 1: Electrical Parameters

This paper discusses the design of an alternative control system based on repetitive feedback for the 3-phase grid connected inverter shown in Fig.1. Stability constraints and trade-off between steady state error and system transient response are analysed. Table 1 shows the electrical parameters of the system.

\section{System Modelling}

The analysis and design of the control system for the voltage source grid connected inverter in Fig. 1 is based upon the 
single phase equivalent circuit shown in Fig.2. The small resistances of inductors and the equivalent series resistance (ESR) of the capacitors are neglected.

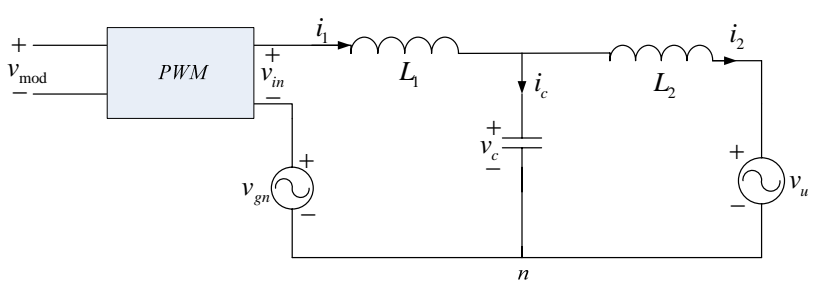

Fig. 2: Single Phase Equivalent Circuit

In Fig. 2, $v_{g n}$ is the voltage difference between the neutral point and middle of the dc link. In control terms this may be viewed as a source of disturbance caused by phase interaction. The disturbance $v_{g n}$ can be expressed by the following equation:

$$
v_{g n}=\frac{v_{a g}+v_{b g}+v_{c g}}{3}
$$

where $v_{a g}, v_{b g}$ and $v_{c g}$ are phase voltages of the phase with respect to the ground. Equation (1) shows that the phase interaction voltage $v_{g n}$ depends on the switching states of all three phases. It can be shown that, when filter capacitors are connected to dc link as shown in Fig.1, the voltage $v_{g n} \approx 0$, showing only a very small switching frequency ripple component [16].

To derive the transfer function of the grid connected inverter we could write the following equations using Kirchhoff's Voltage Law (KVL) and Kirchhoff's Current Law (KCL) based upon Fig. 2.

$$
\begin{aligned}
& v_{\text {in }}-v_{c}=L_{1} \frac{d i_{1}}{d t} \\
& i_{c}=i_{1}-i_{2} \\
& i_{c}=C \frac{d v_{c}}{d t} \\
& v_{c}-v_{u}=L_{2} \frac{d i_{2}}{d t}
\end{aligned}
$$

Based on these equations, we can represent the system using the following block diagram.

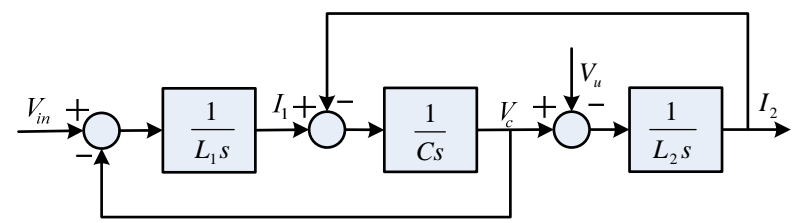

Fig. 3: Block Diagram of the Single-Phase Circuit

\section{Proposed Control Scheme}

The proposed digital controller comprises a conventional two loop feedback system and a repetitive controller, as shown in Fig. 4. The repetitive feedback controller (RC) requires the basic plant (i.e. the two loop system $\left.G_{p}(\mathrm{~s})\right)$ to be stable. Since direct feedback of the output grid current of an LCL filter on its own is inherently unstable, it is necessary to have another feedback loop of the capacitor current or the current in the main inductor $L_{1}[16]$ to stabilize the system. The transfer function relating the output current $I_{2}$ to the reference current $I_{\text {ref }}$ (assuming the PWM block is a unity gain block) can be shown to be,

$$
I_{2}=\frac{G_{c}(s) G_{s}(s)}{1+G_{c}(s) G_{s}(s)} I_{r e f}-\frac{G_{s}(s)}{1+G_{c}(s) G_{s}(s)} D(s)(6)
$$

where, $G_{s}(s)$ is the transfer function of the two loop plant given by,

$$
G_{s}(s)=\frac{1}{\left(L_{1} L_{2} C\right) s^{3}+\left(K_{c} L_{2} C\right) s^{2}+\left(L_{1}+L_{2}\right) s}
$$

and $D(s)$ is the transfer function of input disturbance, which is given by,

$$
D(s)=V_{u}\left(L_{1} C s^{2}+K_{c} C s+1\right)
$$

The system in Fig. 4 can be reduced to that in Fig. 5, with $G_{P}(z)$ given by,

$$
G_{p}(z)=\frac{G_{c}(z) G_{s}(z)}{1+G_{c}(z) G_{s}(z)}
$$

The simplified form of the overall control scheme has been represented by Fig. 5 which will be used for analysis later on.

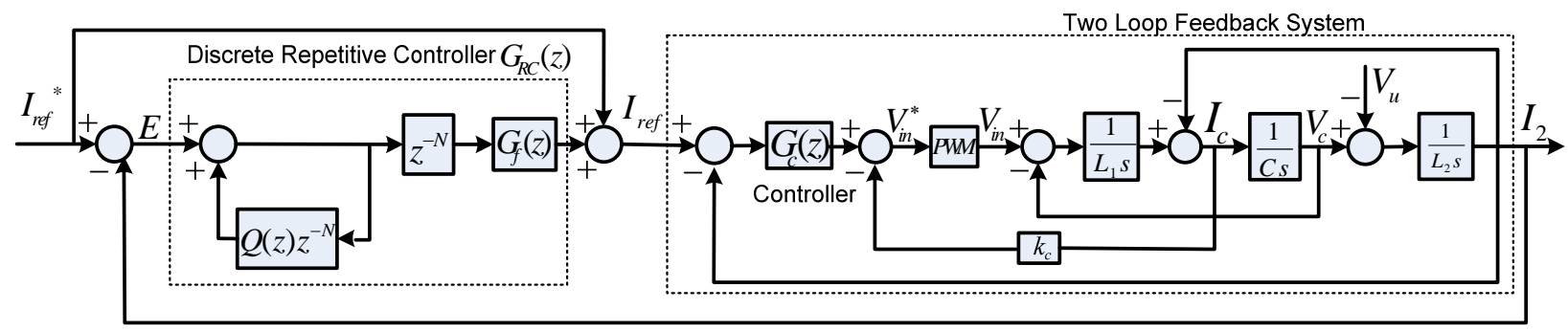

Fig 4: Overall Block Diagram of Proposed Control Scheme 


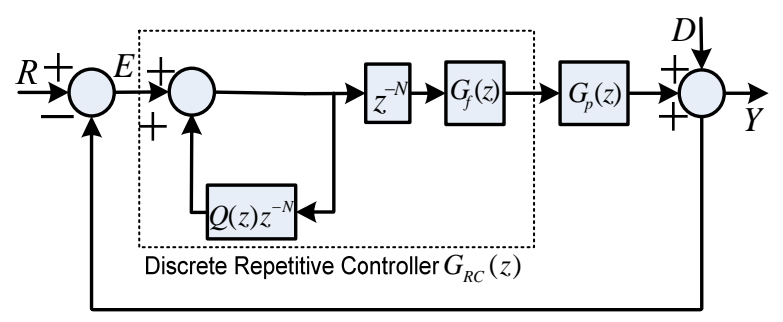

Fig 5: Simplified Block Diagram of Proposed Controller

\subsection{The Conventional Two Loop Feedback System}

We choose the controller to be a simple proportional controller, such that $G_{c}(z)=K_{P}$, since alternative classical controllers such as PID or one of its derivatives, were found to provide marginal improvements (if any) in comparison, at the expense of additional complexity. The values of $K_{p}=6$ and $K_{c}=13$ were selected to provide a compromise between stability, speed of response and disturbance rejection as discussed in [16]. The bode diagram of the two loop system is shown in Fig. 6; the system has a phase margin of $52.6^{\circ}$ and a gain margin of $7.88 \mathrm{~dB}$. The loop gain at $50 \mathrm{~Hz}$ is $18 \mathrm{db}$ and reduces further at higher frequencies. Hence the disturbance rejection at $50 \mathrm{~Hz}$ and its harmonics will be relatively poor. However, it is not possible to increase the loop gain any further as without compromising stability.

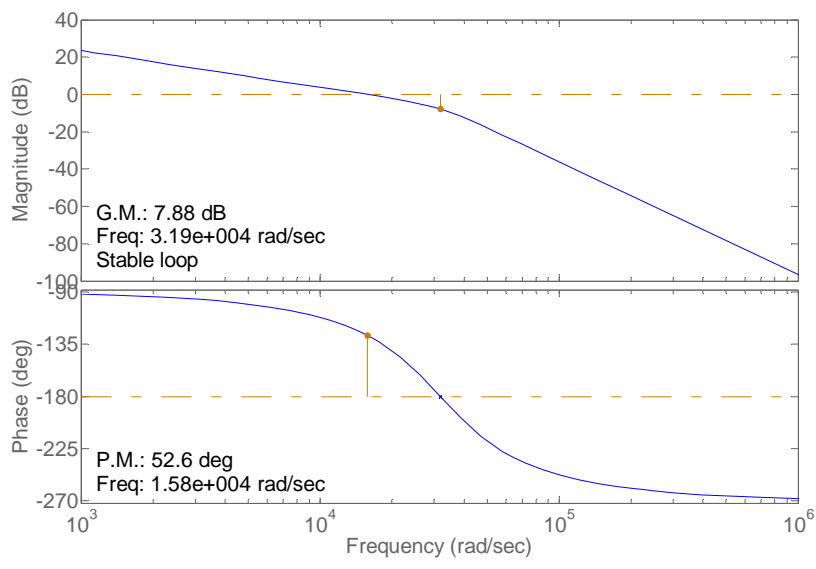

Fig. 6: Bode Plot of Conventional Two Loop Feedback System

\subsection{The Digital Repetitive Controller}

The theory of repetitive control (RC) is based on the internal model principal [17-19], whereby a model of the repetitive reference and disturbance signals is included in the controller. The RC tracks the error on a cycle by cycle basis and corrects the control effort on a periodic basis to compensate for the error.
In the discrete time domain, a periodic signal with a known period $T$ can be generated by a time delay block $z^{-N}$ with a positive feedback loop. Here, $N$ is the number of samples in one period given by,

$$
N=\frac{T}{T_{s}}
$$

where, $T$ is the time period of the any periodic input and $T_{s}$ corresponds to sampling time. Normally $N$ is a large number and hence a basic RC requires a large memory buffers which is one of its drawbacks [20]. The transfer function $G_{R B}(z)$ of a basic RC comprises a gain $K_{R}$ multiplied by the transfer function of a periodic signal generator,

$$
G_{R B}(z)=K_{R} \frac{z^{-N}}{1-z^{-N}}=\frac{K_{R}}{z^{N}-1}
$$

The control objective is to find an appropriate optimal value of the repetitive controller gain $K_{R}$ such that the tracking error converges to zero as the number of iterations approaches to infinity.

$$
\lim _{k \rightarrow \infty}\|e(k)\|=0
$$

The basic repetitive feedback control in (10) is most suitable for those applications where the period $T$ is constant or accurately measureable [20]. This basic repetitive feedback does not ensure stability and error convergence criteria and is normally modified to overcome these problems.

To avoid pure integration, a filter $Q(z)$ is introduced in the basic repetitive control structure, followed by a compensator $G_{f}(z)$ such that

$G_{R C}(z)=G_{R}(z) G_{f}(z)$
where, $G_{R}(z)=K_{R} \frac{z^{-N}}{1-Q(z) z^{-N}}$

The filter $Q(z)$ ensures the stability and robustness of the system. It can be either a low-pass filter or a constant less than 1.

The compensator $G_{f}(z)$ should ideally be designed to be a zero magnitude and phase compensator for the closed loop transfer function of the plant [9]. This however results in a complex compensator, which is computationally costly to implement. A reasonable approximation is achieved by selecting $G_{f}(z)$ to be equal to a gain $K_{R}$ multiplied by the time advance unit $z^{k}[15]$.

$$
G_{f}(z)=z^{k} K_{R}
$$

The time advance $z^{k}$ compensates for the phase lag of the inverter to improve stability. 
Using equations (13) and (15), we can rewrite the transfer function of RC as follows:

$$
G_{R C}(z)=\frac{K_{R} z^{-N+k}}{1-Q(z) z^{-N}}
$$

There are various schemes to design the $Q$-filter and the compensator $G_{f}(z)$ to improve the robustness of RC [21] . In this paper we select $Q$ filter as a constant less than 1 and $k=3$ for the leading unit of the compensator. The value of $K_{R}$ is adjusted after selecting the value of $Q$. The values of $K_{R}$ and $Q$ are tuned to ensure stability while achieving a good speed of response and steady state error.

From Fig. 5, the error $E(z)$ in terms of the reference $R(z)$ and the disturbance $D(z)$ can be derived as:

$$
\begin{aligned}
E(z)= & \frac{\left[1-G_{p}(z)\right]\left[z^{N}-Q(z)\right]}{z^{N}-\left[\left(Q(z)-G_{f}(z) G_{p}(z)\right]\right.} R(z) \\
& +\frac{\left[Q(z)-z^{N}\right]}{z^{N}-\left[\left(Q(z)-G_{f}(z) G_{p}(z)\right]\right.} D(z)
\end{aligned}
$$

Theorem: Assume two systems $G_{1}$ and $G_{2}$ are connected in a feedback loop, then the closed loop system is input-output stable if $\left\|G_{1}\right\| .\left\|G_{2}\right\|<1$

According to the above gain theorem the overall stability conditions can be devised as:

a) The roots of characteristic equation, $1+G_{c}(z) G_{s}(z)=0$ of conventional two loop feedback system without RC should be inside the unit circle.

b) From equation (17),

where,

$$
\left|H\left(e^{j \omega T}\right)\right|<1
$$

$$
H\left(e^{j \omega T}\right)=Q\left(e^{j \omega T}\right)-e^{j \omega T} K_{R} G_{P}\left(e^{j \omega T}\right)
$$

and,

$$
\omega \in\left[0, \frac{\Pi}{T}\right] \text {, and } T=\text { Sample Time. }
$$

\section{Selection of Controller Parameters for Robustness}

The two parameters, $K_{R}$ and $Q$, are closely related to the system stability. The critical value $Q_{\text {critical }}$ of $Q$ at which the system becomes unstable, for a given value of $K_{R}$, was calculated using equation (18) and verified by simulation. The results are plotted in Fig. 8. Fig. 9 illustrates the relationship between the speed of response of the controller and the parameters $Q$ and $K_{R}$. Basically, for a given value $K_{R}$ the speed of response improves by increasing $Q$, at the expense of reducing stability. Increasing $K_{R}$ improves the steady state error (SSE) for a given value of $Q$. Using Figs. 7 and 8, the values of $Q$ and $K_{R}$ can be selected to ensure stability and achieve a fast speed of response and small steady state error. $Q=0.9$, and $K_{R}=0.4$ were found to give a satisfactory performance.

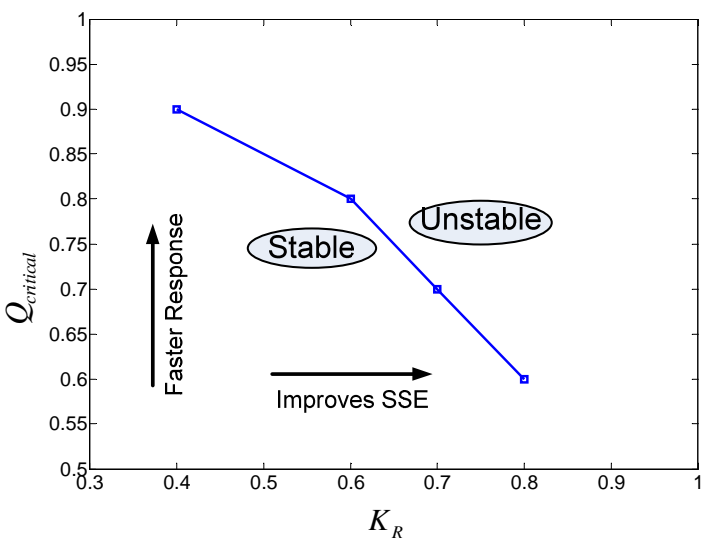

Fig. 7: Relationship between $Q_{\text {critical }}$ and $K_{R}$

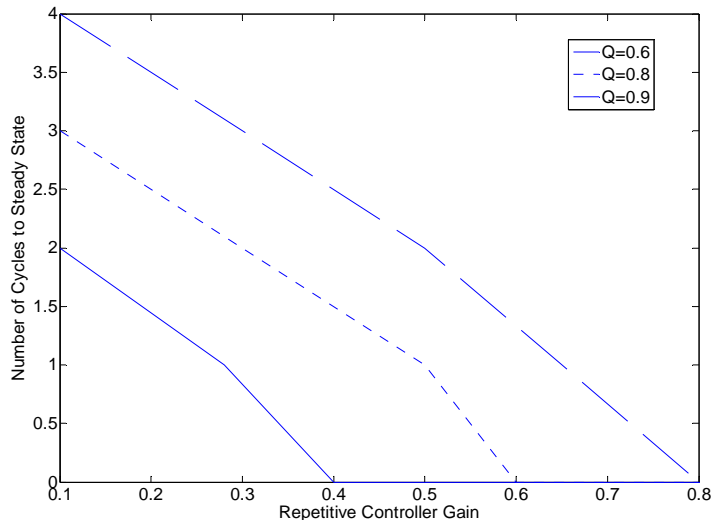

Fig 8: Graph between Number of Cycles to SSE and $K_{R}$

The value of the inductor $L_{2}$, which is determined by the grid impedance, can vary significantly depending on the site where the inverter is installed. This uncertainty needs to be taken into account to ensure that the system remains stable under the worst condition. To assess the robustness of the system to the uncertainty in the value of $L_{2}$ the bode diagram of the system including the RC was plotted for different values of $L_{2}$ as shown in Fig. 9. As it can be seen, the effect of $L_{2}$ on system stability and gain at the fundamental frequency and its harmonics is negligible, thus illustrating the robustness of the system to variations in the value of $L_{2}$. This was also verified by simulation. 


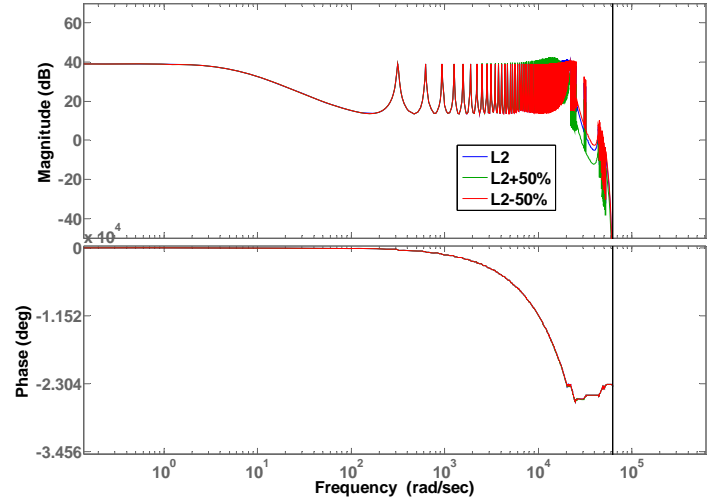

Fig 9: Bode diagram of the system including RC illustrating robustness to variation in $L_{2}$

\section{Simulation Results}

Detailed simulation has been carried out using the MATLAB Simpower Systems Toolbox. The system parameters are shown in Tables 1 and 2. Four cases with different utility THD values have been considered; the grid harmonic content when the THD was $14 \%$ is shown in Table 3 . The reference current was $100 \mathrm{~A}$ (peak).

\begin{tabular}{|l|c|}
\hline \multicolumn{1}{|c|}{ Parameter } & Value \\
\hline Outer Loop Controller Gain $K_{P}$ & 6 \\
\hline Inner Loop Capacitor Gain $K_{C}$ & 13 \\
\hline Repetitive Controller Gain $K_{R}$ & 0.4 \\
\hline Value of $Q$ & 0.9 \\
\hline$N$ for delay term $z^{-N}$ & 400 \\
\hline
\end{tabular}

Table 2: Controller Parameters

\begin{tabular}{|l|c|c|c|c|c|c|}
\hline $\begin{array}{l}\text { Harmonic } \\
\text { Number }\end{array}$ & $3^{\text {rd }}$ & $5^{\text {th }}$ & $7^{\text {th }}$ & $9^{\text {th }}$ & $11^{\text {th }}$ & $13^{\text {th }}$ \\
\hline $\begin{array}{l}\text { Fundamental } \\
\text { Component }\end{array}$ & 35 & 25 & 15 & 5 & 2 & 1 \\
\hline
\end{tabular}

Table 3: Grid voltage harmonics when the THD is $14 \%$

Fig. 11 shows the output current without the use of repetitive controller, while Fig. 12 shows simulation results with the repetitive controller. The THD of the output current improves significantly in any case when the RC is used. For example, in the $2^{\text {nd }}$ case when the utility THD is $14 \%$, the output current THD with the repetitive controller improves from $9.5 \%$ to $4.4 \%$.

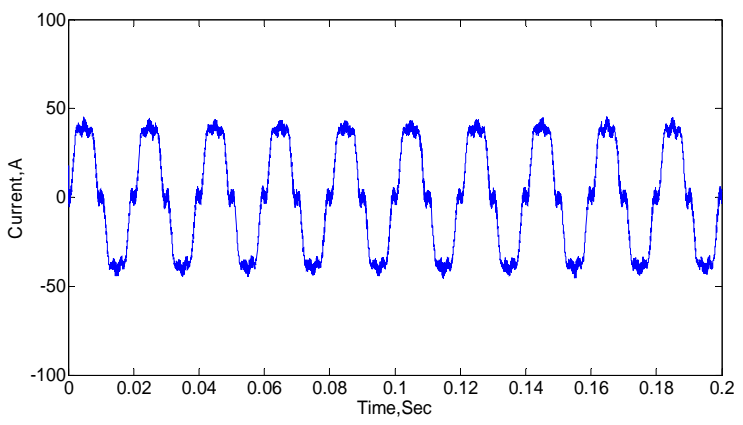

Fig 11: Output Current without RC

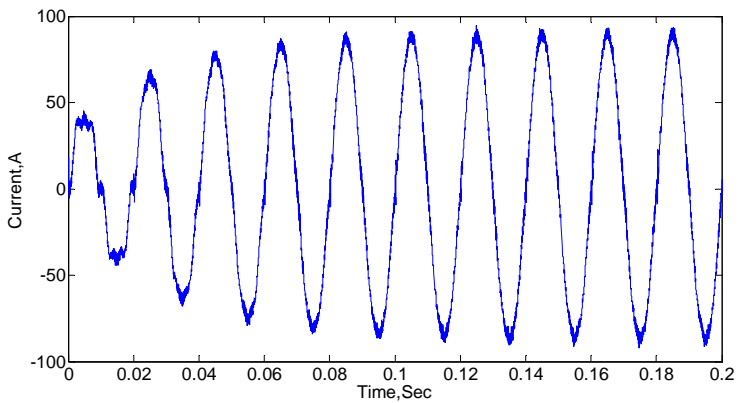

Fig 12: Output Current with RC When Utility THD $=14 \%$ and $Q=0.9, K_{R}=0.4$

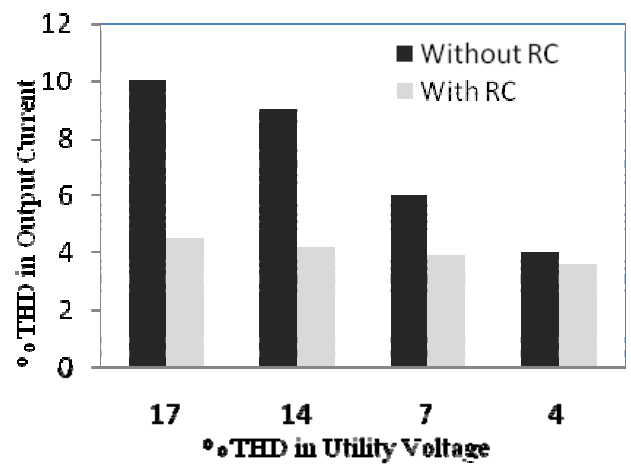

Fig 13: Comparison of \% THD in Output Currents at Different Utility THD levels with and without RC

\section{Conclusion}

Simulations results show repetitive control can significantly improve the THD quality of the output current. The RC parameters need to be selected carefully to ensure stability despite uncertainty in grid impedance, while achieving a fast response and a small steady state error. The proposed controller was demonstrated to be robust to changes in grid impedance. Further work is needed to improve the steady state error.

\section{Acknowledgements}

Mr. M. Jamil is thankful to National University of Science and Technology (NUST), Pakistan for giving scholarship for 
his PhD studies. Also thanks to Dr. Wali Mohammed Trust for partial support.

\section{References}

[1] T. S. Basso and R. DeBlasio, "IEEE 1547 series of standards: interconnection issues", IEEE Transactions on Power Electronics, vol. 19, pp. 1159-1162, (2004).

[2] "Engineering Recommendation G59/1, 'Recommendations for the Connection of Embedded Generating Plant to the Regional Electricity Companies' Distribution Systems", Electricity Association (Engineering Services), (1991).

[3] B. Qu, X.-y. Hong, W.-X. Yao, Z.-y. Lu, and J. M. Guerrero, "An Optimized Deadbeat Control Scheme Using Fuzzy Control in Three-Phase Voltage Source PWM Rectifier", Twenty-Fourth Annual IEEE Conference on Applied Power Electronics Conference and Exposition (APEC), pp. 1215-1219, (2009).

[4] Kui-Jun Lee, Nam-Ju Park, and D.-S. Hyun., "Optimal current controller in a three-phase grid connected inverter with an LCL filter", Hong Kong Conference on Control, (2007).

[5] I. J. Gabe, J. R. Massing, V. F. Montagner, and H. Pinheiro, "Stability analysis of grid-connected voltage source inverters with LCL-filters using partial state feedback", 12th European Conference on Power Electronics and Applications, pp. 1-10, (2007).

[6] I.-S. Kim, "Sliding mode controller for the singlephase grid-connected photovoltaic system", Applied Energy, vol. 83, pp. 1101-1115, (2006).

[7] A. V. Timbus, M. Ciobotaru, R. Teodorescu, and F. Blaabjerg, "Adaptive resonant controller for gridconnected converters in distributed power generation systems", Twenty-First Annual IEEE Conference on Applied Power Electronics and Exposition(APEC), p. 6 ,(2006).

[8] T. Erika and D. G. Holmes, "Grid current regulation of a three-phase voltage source inverter with an LCL input filter", IEEE Transactions on Power Electronics, vol. 18, pp. 888-895, (2003).

[9] S. Chen, Y. M. Lai, S. C. Tan, and C. K. Tse, "Analysis and design of repetitive controller for harmonic elimination in PWM voltage source inverter systems", IET Journal of Power Electronics, vol. 1, pp. 497-506, (2008).

[10] G. Weiss, Z. Qing-Chang, T. C. Green, and L. Jun, "H infinity repetitive control of DC-AC converters in microgrids", IEEE Transactions on Power Electronics, vol. 19, pp. 219-230, (2004).

[11] W. Wang, S. K. Panda, and X. Jian-Xin, "Control of high performance DC-AC inverters using frequency domain based repetitive control", International Conference on Power Electronics and Drives System ( PEDS),pp. 442-447,(2005).
[12] S. M. Sharkh, M.Abu-Sara, and Z. F. Hussein., "Current control of utility -connected DC-AC threephase voltage-source inverters using repetitive feedback", European Conference on Power Electronics and Applications, (EPE), p. 6, (2001).

[13] Z. Qing-Chang, T. Green, L. Jun, and G. Weiss, "Robust repetitive control of grid-connected DC-AC converters", 41st IEEE Conference on Decision and Control, vol.3, pp. 2468-2473,(2001).

[14] S. PengFei, J. Xiong, Z. Kai, and Z. Liang, "One cost-effective feedback control scheme for PWM inverters based on repetitive control", $1^{\text {st }} I E E E$ Conference on Industrial Electronics and Applications (ICIEA), pp. 1-5,(2006).

[15] L. Fei, D. Shanxu, X. Pengwei, C. Guoqiang, and L. Fangrui, "Design and control of three-phase PV grid connected converter with LCL filter", 33rd Annual Conference of the IEEE Industrial Electronics Society, (IECON), pp. 1656-1661,(2007).

[16] S. M. Sharkh and M.Abu-Sara, "Digital current control of utility connected two-level and three-level PWM voltage source inverters", European Journal of Power Electronic, vol. 14-4, (2004).

[17] Yigang Wang, Danwei Wang, and X. Wang, "A three-step deisgn method for performance improvement of robust repetitive Control", American Control Conference, (2005).

[18] Z. Keliang and W. Danwei, "Digital repetitive learning controller for three-phase CVCF PWM inverter", IEEE Transactions on Industrial Electronics, vol. 48, pp. 820-830, (2001).

[19] Z. F. Hussein, "Current Control of Three-Phase PWM Inverter for Flywheel Energy Storage System", PhD Thesis , University of Southampton, (2000).

[20] G. Pipeleers, B. Demeulenaere, J. De Schutter, and J. Swevers, "Generalised repetitive control: relaxing the period-delay-based structure", IET Journal of Control Theory and Applications, vol. 3, pp. 15281536, (2009).

[21] Y. Wang, F. Gao, and F. J. Doyle Iii, "Survey on iterative learning control, repetitive control, and runto-run control", Journal of Process Control, vol. 19, pp. 1589-1600, (2009). 